\title{
MCT1 genetic polymorphism influence in high intensity circuit training: A pilot study
}

\author{
Rocío Cupeiro ${ }^{\text {a }}$, Pedro J. Benito ${ }^{\text {a }}$, Nicola Maffulli ${ }^{\mathrm{c}, *}$, F. Javier Calderón ${ }^{\mathrm{a}}$, \\ Domingo González-Lamuño ${ }^{b}$ \\ ${ }^{2}$ Laboratory of Exercise Physiology. Department of Health and Human Performance, Universidad Politecnica de Madrid. Madrid. Spain \\ ${ }^{\mathrm{b}}$ Laboratory of Puediatries. School of Medicine-IFIMAV, Universidad de Cantabria-Hospital M. Vuldecilla. Santander. Spain \\ ' Queen Mary University of London, Barts and The London Sthool of Medicine and Dentistry, Centre for Sports and Exercise Medicine. \\ Mile End Hospital, London, United Kingdom
}

\begin{abstract}
Monocarboxylate Transporter 1 (MCT1) mediates the transport of the main fraction of lactate across the sarcolemma. A common polymorphic MCT1 variant has been identified, but its role in high intensity exercise performance has not been defined. We investigated the influenee of MCT1 A1470T polymorphism (rs1049434) on lactate accumulation alter high intensity circuil training. Ten men aged 20-26 performed three controlled circuil training (CWT) sessions at $60 \%, 70 \%$, and $80 \%$ of the 15 repetition maximum (15 RM), in non-conseculive days. CWT included three sets of a circuit of eight exercises, obtaining lactate measurements immediately after each set had been completed. Two independent variables were analysed: MTC1 genotypes according to the presence or absence of the A1470T polymorphism, and the intensity of circuit training. Genotype distributions were in Hardy-Weinberg equilibrium, being $30 \%$ wild-type, $50 \%$ heterozygotes, and $20 \%$ mutated homozygotes. Mean lactate concentration at $80 \%$ of $15 \mathrm{RM}$ were significantly higher than the mean lactate values at the other intensities $(p<0.01)$. Significant dilferences between genetic groups were found in the lactate accumulation slope at $80 \%$ of $15 \mathrm{RM}(p=0.02)$ and in the maximal lactate concentration reached by all subjects in the study $\left(L_{\max }\right)(p=0.03)$. The carriers of the A1470T polymorphism in the MTC1 gene seem to exhibit a worse lactate transport capability into the less active muscle cells for oxidation.
\end{abstract}

Keywords: Monocarboxylate transporters; Lactate accumulation; Circuil weight training; A1470T polymorphism

\section{Introduction}

Lactate transport across the sarcolemma is mainly mediated by proton-linked monocarboxylate transporters (MCT 1 and MCT4). ${ }^{1-4}$ In rats, there is a high correlation between MCT1 expression in muscle and the rate of blood lactate elcarance. ${ }^{5.6}$ In humans, expression of MCT1 may correlates with lactate removal ${ }^{7,8}$ and with the amount of lactate oxidised into muscle cells. ${ }^{9}$ In humans, defects in MCT1 transporter have been associated with several pathologies. Symptoms and signs of muscle injury on exercise and heat exposure plus subnormal erythrocyte lactate transport have been related with the presence of two mutations in

\footnotetext{
* Corresponding author.

E-mail address: n.maffulli@qmul.ac.uk (N. Maffulli).
}

the gene that codifies MCT1 ${ }^{10}$ An Al470T polymorphism (rs 1049434) in the MCT1 gene (resulting in a glutamic acid to-aspartic acid change in codon 490), whose physiological role is still indeterminate, has been also reported. ${ }^{10}$ Although there appear to be no clinical effects of this polymorphism, a $40 \%$ reduction of lactate transport rate has been identified in the erythrocytes of both individuals homozygous and heterozygous for this polymorphic variant. ${ }^{10}$

In high-intensity exercise, anaerobic metabolism accounts for the subsequent lactic accumulation, ${ }^{11}$ especially in resistance training, ${ }^{12,13}$ making it relevant to study this metabolite and its movement in the body during strength training.

We performed a pilot study to investigate the influence of the A1470T polymorphism (rs1049434) MCT1 genotypes on lactatc accumulation after circuit training. Wc also examined whether the effect of this polymorphism on lactate accumu- 
lation depends on the intensity of the circuit training. We hypothesised that the A 1470T gene polymorphism might lead to a reduction in the function of the MCT1 transporter, which would be reflected in a greater blood lactate accumulation.

\section{Methods}

Ten men $(23.6 \pm 2.2$ years; body mass: $78.2 \pm 7.0 \mathrm{~kg}$; height: $179.2 \pm 6.3 \mathrm{~cm}$ ) gave their written informed consent to participate in this study, which was approved by the Human Research Review Ethics Committee of the School of Sport Sciences, Universidad Politecnica de Madrid. All had at least 1 year of experience in resistance training for 3-5 h per week, were between 20 and 30 years old, physically active and non-smokers. Individuals with diabetes, cardiovascular disease, or other metabolic disorders were excluded from the study.

Percentage body fat was estimated from six skinfolds ${ }^{\mathbf{1 4}}$. triceps, subscapular, suprailium, abdominal, calf, and thigh. The following formula were applied:

\%Body fat $=\left(\left(\left(\sum 6\right.\right.\right.$ skinfolds $\left.\left.) \cdot 0.097\right)+3.64\right) \times 100^{-1}$

Body mass and height were also measured. ${ }^{14}$ Muscle mass (MM) was estimated using Martin's equation ${ }^{15}$.

MM $(g)=$ height $[0.0553 \cdot($ thigh circumference

- thigh skinfold) $2+0.0987$

(forearm circumference) $\cdot 2+0.0331$

(calf circumference - calf skinfold) - 2] - 2445

The 15 repetition maximum ( $15 \mathrm{RM}$ ) for each exercise included in the circuit weight training (CWT) protocol was assessed twice on different days during the two wks prior to the CWT sessions, with an intra-class correlation coefficient of 0.870 . The subjects came to the laboratory in non-consecutive days, assessing each day one of the following exercise groups: leg press and shoulder press; leg extensions, cable triceps extensions and lat pull-down; leg curl, biceps curl and seated chest press. A standardised warmup was performed for $10 \mathrm{~min}$ before each assessment, ${ }^{16}$ and all the repetitions were performed at a pre-set rhythm of $I s$ for the concentric phase and $2 \mathrm{~s}$ for the eccentric phase. The sounds of a pre-recorded CD ROM indicated this rhythm to the subjects. A movement specific warm-up was performed, with three sets of 15 repetitions (at $50 \%, 70 \%$, and $90 \%$ of the estimated $15 \mathrm{RM}$ ) with $2 \mathrm{~min}$ of recovery between the sets. After 5 min of recovery, a inal set of 15 repetitions was carried at $100 \%$ of the estimated 15 RM. If the subject was able to perform more than 15 repetitions, another set was performed after $5 \mathrm{~min}$, increasing the load of the $15 \mathrm{RM}$ of $2.5 \%$. The load was lowered $(-2.5 \%$ of $15 \mathrm{RM})$ if the subject could not perform 15 repetitions. A maximum of two attempts were performed in the same day. ${ }^{17}$ During the tests, all the subjects were verbally encouraged in a standardised fashion to perform as many repetitions as possible.

Participants came to the laboratory in three nonconsecutive days to undergo a CWT session at $60 \%, 70 \%$ or $80 \%$ of $15 \mathrm{RM}$ in a random order. Each session included three circuits of eight exercises (seated chest press, leg extensions, lat pull-down, leg press, shoulder press, leg curl, biceps curl, and cable triceps extensions), performing 15 repetitions in each exercise. Recoveries of 5 min between circuits and $10 \mathrm{~s}$ between exercises were set. Before each session, the subject performed a warm up circuit at $20 \%$ of their $15 \mathrm{RM}$. Throughout the session, the rhythm at which the exercises were performed was the same as the one used in the testing session, and given by the same CD ROM used in the preliminary session.

All of the subjects completed an initial adaptation phase, prior to all the testing. This involved the same CWT used in the testing sessions, performed three times per week during 4 weeks. All the exercises were performed on machines (Panatta, Italy). No free weights were used.

Capillary blood samples were obtained immediately after each circuit had been completed, and at minutes 3 and 5 after the end of the exercise bout. These samples were analysed by enzymatic method using the YSI 1500 Sport Analyzer (YSI, Yellow Springs, OH, USA), obtaining the lactate concentrations immediately after the first (Lc1), the second (Lc2), and the third circuit (Lc3), and at minutes 3 and 5 after the end of the exercise bout. Calibrations before and after each session were carried according to manufacturer's recommendations.

Slopes of lactate accumulation (LA) throughout the sessions were obtained by individual linear regression equations. First, lactate values were plotted versus time of sampling for each subject and intensity. Then, a regression of the line obtained was performed, calculating its linear regression equation. Data of the nine subjects who completed the $60 \%$ and $70 \%$ sessions, and at least two circuits of the $80 \%$ session were included for this calculation.

To obtain the mean lactate concentration at each intensity $(60 \%, 70 \%$ and $80 \%)$, lactate concentrations measured immediately after each of the three circuits were averaged ( $L_{\text {average }}$ ). For every subject, the maximal lactate concentration reached throughout the whole study is reported $\left(L_{\max }\right)$, as well as the maximal lactate concentration reached at each intensity ( $L_{\max }$ Int).

Genomic DNA was extracted from peripheral blood using a QIAamp DNA Blood Mini kit (QIAGEN, Hilden, Germany). Genomic DNA from the subjects was analysed by polymerase chain reaction (PCR) amplification of a fragment containing the A1470T polymorphism of the MCT1 gene (rs1049434, exon 5) and following direct sequencing. The primers used for amplification were as follows ${ }^{10}$ : sense primer 5'-ACA CAT ACT GGG CAT GTG GC-3' (1455-1474); antisense primer 5'-AAA TCC CAT CAA TGA ACA ACT GGT ATG ATT TCC AC-3' (1807-1841). PCR reaction was made in a total volume of $50 \mu \mathrm{L}$ containing: $3 \mu \mathrm{L}$ genomic DNA, $1.5 \mathrm{mM} \mathrm{MgCl} 2,0.2 \mathrm{mMdNTP}$ mix, 
$0.4 \mu \mathrm{M}$ primer, $4 \%$ dimethyl sulfoxide (SIGMA) and I U Taq polymerase (BioTaq Polimerase, BioLine, London, UK). The amplification consisted of initial denaturation $\left(94^{\circ} \mathrm{C}, 5 \mathrm{~min}\right)$; 35 cycles consisting of denaturation $\left(94^{\circ} \mathrm{C}, 1 \mathrm{~min}\right)$, annealing $\left(55^{\circ} \mathrm{C}, 1 \mathrm{~min}\right)$, and extension $\left(72^{\circ} \mathrm{C}, 1 \mathrm{~min}\right)$; and final exten$\operatorname{sion}\left(72^{\circ} \mathrm{C}, 10 \mathrm{~min}\right)$. PCR products were electrophoresed on $1.5 \%$ agarose gel to verify successful amplification of the 387 bp fragments. Prior to sequencing, the PCR products were purified using QIAquick PCR Purification Kit (QIAGEN, Hilden, Germany). The sequencing reactions were carried out using dRhodamine Terminator Cycle Sequencing Kit (Applied Biosystems, Foster City, CA, USA), and analysed on the automated ABI Prism 310 Genetic Analyzer (Applied Biosystems, Foster City, CA, USA). The nucleotide sequence of exon 5 was obtained from GeneBank (accession: NM_003051).

Subjects were allocated to two groups according to their profile of the MCT1 gene. Subjects who presented the wild-type in their two alleles (AA) were allocated in the non-carriers group (NC), and the subjects who presented the A1470T polymorphism in at least one of their two alleles (AT or TT) were allocated in the carriers group (C).

Data are presented as mean \pm standard deviation (SD). Chi-square analysis was used to test for Hardy-Weinberg equilibrium. Given the sample size and the variables distribution, non-parametric tests were used. Two independent variables were examined: $\mathrm{MTCl}$ genotype according to the presence or absence of the A1470T polymorphism, and CWT intensities. Mann Whitney U tests were performed to analyse the differences between carrier (C) and non-carrier (NC) of the polymorphism for all the lactate variables at each intensity. The mean values for each intensity were compared using Friedman ANOVA's for repeated measures from the same subjects, followed by Wilcoxon test comparisons. An $n$ of 10 in each group was adequate for a 0.05 alpha level and provide a statistical power of 0.80 .

\section{Results}

The genotype distribution ( $30 \%$ wild-type, $50 \%$ heterozygotes, $20 \%$ mutated homozygotes) showed Hardy-Weinberg equilibrium. The allelic frequency was 0.55 and 0.45 for the wild-type allele and for the polymorphic allele, respectively.

The carriers were $24.1 \pm 1,3$ year old, had a body mass of $81.2 \pm 6.7 \mathrm{~kg}$, and were $180.2 \pm 5.1 \mathrm{~cm}$ tall. The non-carriers were $23.7 \pm 3.2$ year old, had a body mass of $71.2 \pm 2.5 \mathrm{~kg}$, and were $173.6 \pm 4.2 \mathrm{~cm}$ tall. There were no differences in the various anthropometric variables, except for body mass $(p=0.03)$.

The mean lactate concentration at $80 \%$ was significantly higher that the mean values at the other intensities $(p<0.01)$ (Table 1).

The Mann-Whitney test showed significant differences in the LA slope at $80 \%$ of 15 RM between the carriers and non-
Table 1

Main lactate variables measured at $60 \%, 70 \%$ and $80 \%$ of $15 \mathrm{RM}$.

\begin{tabular}{lccc}
\hline & $60 \%(n=10)$ & $70 \%(n=10)$ & $80 \%(n=9)$ \\
\hline Lcl $\left(\right.$ mmol L $\left.^{-1}\right)$ & $7.1 \pm 2.4$ & $8.7 \pm 3.2$ & $12.2 \pm 3.2$ \\
Lc2 $\left(\mathrm{mmol} \mathrm{L}^{-1}\right)$ & $9.5 \pm 3.7$ & $11.0 \pm 4.2$ & $16.8 \pm 3.7$ \\
$\mathrm{Lc} 3\left(\mathrm{mmol} \mathrm{L}^{-1}\right)$ & $11.3 \pm 3.9$ & $13.0 \pm 4.3$ & $19.9 \pm 5.0$ \\
$L_{\text {average }}\left(\mathrm{mmol}^{-1}\right)$ & $9.3 \pm 3.2$ & $10.9 \pm 3.6$ & $15.9 \pm 3.6^{*}$ \\
$L_{\max }$ Int $\left(\mathrm{mmol} \mathrm{L}^{-1}\right)$ & $11.5 \pm 3.8$ & $13.8 \pm 4.7$ & $19.5 \pm 4.7$ \\
\hline
\end{tabular}

Values are means \pm SD. Lcl. lactate concentration immediately after the first lap. Lc2, lactate concentration immediately after the second lap. Lc3, lactate concentration immediately after the thitd lap. $L_{\text {average }}$ average of Lc1, Lc2 and $L C 3 . L_{\max }$ Int, maximal lactate concentration observed at each intensity.

${ }^{*}$ Significant differences with the other intensities, $p<0.01$.

carriers $(p=0.02)$. Fig. 1 shows the values for the LA slopes at the three exercise intensities.

The test also detected significant differences for $L_{\max }$ $(p=0.03)$. $L_{\max }$ occurred after the last circuit at the highest intensity they could complete ( $70 \%$ or $80 \%$ of 15 RM) in all but two subjects. One of them reached it after the last $60 \%$ CWT, and the other at minute 3 after the end of the $80 \%$ CWT. The values of the main lactate variables for both genetic groups are shown in Table 2.

\section{Discussion}

We found significant differences between genetic groups in the LA slope produced during the $80 \%$ CWT, and also in the highest lactate concentration value reached during the study.

Merezhinskaya et al. first described the A1470T polymorphism of MCT1 in patients with deficiency of lactate transport. ${ }^{10}$ There was no clinical relevance of the polymor-

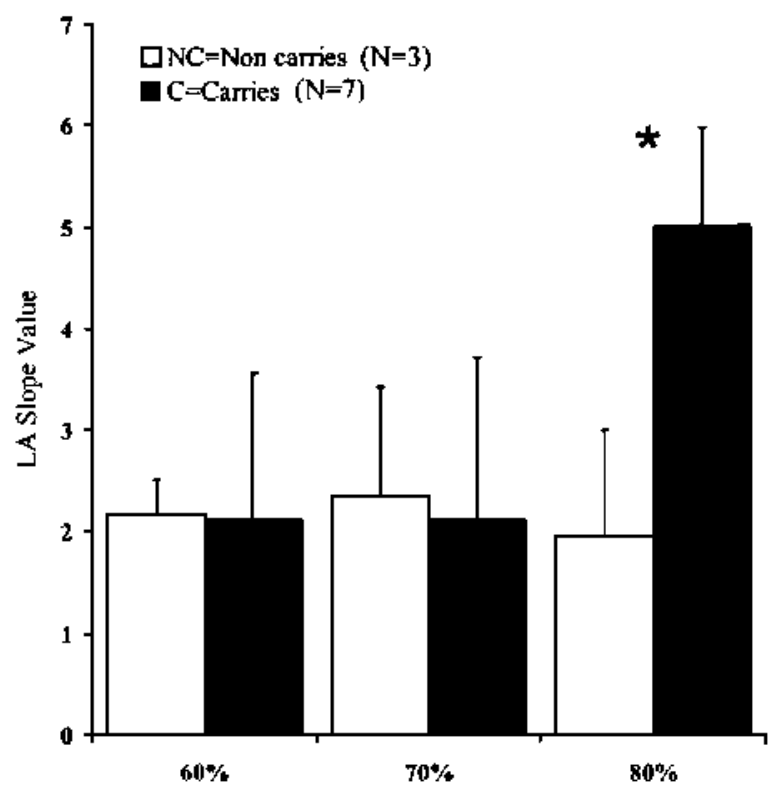

Fig. 1. Values of the slopes for the lactate accumulation at $60 \%, 70 \%$ and $80 \%$ of $15 \mathrm{RM}$. "Significant differences with the other intensities, $p=0.03$. 
Table 2

Values of the main lactate variables for non-cartiers and cartiers.

\begin{tabular}{|c|c|c|c|}
\hline & & $\mathrm{C}$ & $\mathrm{NC}$ \\
\hline \multirow{2}{*}{$60 \%$} & $L_{\text {average }}\left(\operatorname{monol} \mathrm{L}^{-1}\right)$ & $8.8 \pm 3.2$ & $10.5 \pm 3.4$ \\
\hline & $L_{\text {nax }}$ Int (mmol L $\left.{ }^{-1}\right)$ & $11.0 \pm 4.1$ & $12.8 \pm 3.1$ \\
\hline \multirow{2}{*}{$70 \%$} & $L_{\text {average }}\left(\right.$ monol $\left.\mathrm{L}^{-1}\right)$ & $10.9 \pm 4.0$ & $10.9 \pm 3.1$ \\
\hline & $L_{\operatorname{nax}}$ Int $\left(\mathrm{mmol} \mathrm{L}^{-1}\right)$ & $14.0 \pm 5.2$ & $13.3 \pm 4.4$ \\
\hline \multirow{2}{*}{$80 \%$} & $L_{\text {average }}\left(\mathrm{mmol} \mathrm{L}^{-1}\right)$ & $16.7 \pm 4.1$ & $14.3 \pm 1.7$ \\
\hline & $L_{\text {nax }}$ Int (mmol L $\left.{ }^{-1}\right)$ & $21.3 \pm 4.7$ & $15.8 \pm 1.9$ \\
\hline \multicolumn{2}{|c|}{$L_{\max }\left(\mathrm{mmol} \mathrm{L}^{-1}\right)$} & $21.3 \pm 4.1$ & $16.3 \pm 2.4$ \\
\hline
\end{tabular}

Values are means $\pm S D, C$, carriers group (heterozygotes plus nutated homozygotes). NC., non-carriets group (homozygotes wild-type). Laverage. average of the lactate concentrations immediately aftet each lap. $L_{\max }$ Int. maximal lactate concentration observed at each intensity. $L_{\max }$. maximal lactate concentration observed in the whole study.

" Significant differences between genetic groups, $p=0.03$.

phism, but erythrocyte lactate transport rates were $60-65 \%$ of normal. We documented a significant difference in the lactate accumulation in subjects undergoing maximal effort performing high intensity circuit training.

Previous investigations in humans have shown that MCT expression is upregulated after physical training. Endurance, ${ }^{18,19}$ sprint, $^{20,21}$ and resistance training ${ }^{22,23}$ increase from $18 \%^{18}$ to $90 \%^{19}$ the amount of MCT1 in skeletal muscles. Even one exercise session can produce variable changes in MCTs expression, ${ }^{7,24}$ depending on the different timing of the assessment of MCTs expression, with decrease of MCTs expression immediately after the exercise session, ${ }^{24}$ or increase several days after the exercise bout. ${ }^{7}$ We have considered this confounding factor selecting our cohort from individuals with similar training experience, and including an identical initial adaptation phase for all the subjects.

One of the missense mutations described by Merezhinskaya et al. ( $A>T$ at coding base 1470) was found in 40 healthy volunteers, and this polymorphism probably had no clinical effect on lactate transport. ${ }^{10}$ In our study, we found this polymorphism in healthy individuals, with an allelic frequency of 0.45 .

The main differences in the interpretation of Merezhinskaya et al. ${ }^{10}$ observations and ours concern the different ways used to assess the MCTI function as lactate transporter. They measured erythrocyte lactate transporter in vitro, and also with an analytic forearm test, the lactate ammonia exercise ratio (LAER) test, modified from the clinical ischemic forearm test. In the LAER test, the blood flow through the exercising arm is controlled to permit diffusion equilibrium between blood and muscle lactate. Despite this control of blood flow, the test and the in vitro assay do not reproduce the conditions occurring during intense physical exercise, where the less active muscles use the lactate as fuel and the muscle lactate transporters (MCT1 and MCT4) operate together. ${ }^{25}$

We used high intensity CWT to produce high lactate concentrations. We expected that the lactate concentration slope would not have been influenced by the training status of our subjects or by their muscle mass, as opposed to absolute values of lactate concentration. The higher concentrations observed in carriers at the end of the CWT session and the higher $L_{\max }$ could be caused by impaired functionality in MCT1 which would lead to impaired lactate transport into the less active muscle cells for oxidation, thus increasing blood lactate concentration. ${ }^{4.5}$ This could increase muscle fatigue, given the results of previous studies showing a negative correlation between MCT1 content and fatigue indices. ${ }^{*}$ The change in the transporter also could increase the risk of suffering similar symptoms as those presented in MCT1 mutations carriers, ${ }^{10}$ especially in conditions such as very intensity training. In our study, the differences between the MCT1 groups occur only at the highest intensity. Trying to explain this lack of difference at lower intensities, we suggest a lactate concentration threshold necessary to observe differences between the groups. Under this threshold, the polymorphic transporter could cover the required lactate movement, but, if the production lactate increases disproportionately, the ability of the modified transporter would be insufficient.

To our knowledge, this is the first study investigating the potential influence of MCTI A1470T polymorphism during progressively more intense exercise. Potential confounders could be the different diet of the subjects, which can influence substrate utilization and thus lactate production, ${ }^{26-28}$ the relatively small, though statistically appropriate, sample size, the different percentages of muscle fibers among the subjects, which determine MCT1 expression, ${ }^{23}$ or the fact that we measured blood lactate instead of muscle lactate concentration.

In conclusion, carriers for MCT1 Al470T polymorphism showed higher lactate accumulations than non-carriers during high intensity CWT. Although this observation should be considered with caution given our small sample size, these results should be taken into account in future studies relating MCT1 expression and lactate transport within exercise. ${ }^{23}$ Further research is also required to investigate the implications of this genetic factor in acid-base balance during exercise, and whether these influences are also present in women.

\section{Practical implications}

- Carriers of the MCT1 A1470T polymorphism have higher lactate accumulation than noncarriers during high intensity CWT.

- This difference in lactate accumulation manifests at high intensity exercise.

- High intensity resistance training loads could be titrated according to the genetic makeup of individual athletes. 


\section{Conflict of interest}

The authors have no financial conflict of interest and no external financial support.

\section{References}

1. Bonen A. The expression of lactate transporters (MCTl and MCT4) in heat and nuscle. Eur J Appl Physiol 2001;86(Novenber (1)):6-11.

2. Halestrap AP, Price NT. The proton-linked monocarboxylate transporter (MCT) family: structure, function and tegulation. Biochem $J$ 1999;343(October (Pt 2)):281-99.

3. Juel C. Current aspects of lactate exchange: lactate $/ \mathrm{H}+$ transpot in human skeletal muscle. Eur J Appl Physiol 2001;86. November (1)):12-6.

4. Juel C. Halestrap AP. Lactate transport in skeletal muscle-role and regulation of the monocatbox ylate transpoiter. J Physiol 1999;517 (June (Pt 3)):633-42.

5. McCullagh KJ, Poole RC. Halestrap AP, et al. Role of the lactate transporter (MCT1) in skeletal muscles. Am J Physiol 1996;271(July (1 Pt 1)): $\mathrm{E} 143-50$.

6. McCullagh KJ, Poole RC., Halestrap AP, et al. Chronic electrical stimulation incteases MCT1 and lactate uptake in ted and white skeletal nuscle. Am J Physiol 1997;273(August (2 Pt 1)):E239-46.

7. Green H. Halestrap A, Mockett $C$. et al. Increases in nuscle MCT are associated with reductions in muscle lactate after a single exercise session in humans. An J Physiol Entocrinol Metab 2002;282(January (1)): El54-60.

8. Thomas C, Perrey S. Lambert K. et al. Monocarboxylate transporters. blood lactate removal after supramaximal exercise, and fatigue indexes in humans. J Appl Physiol 2005;98(March (3)):804-9.

9. Bonen A, Tonouchi M. Miskovic D. et al. Isoform-specific regulation of the lactate transporters MCTl and MCT4 by contractile activity. $A m$ $J$ Physiol Endocrinol Metab 2000;279(November (5)):E1131-8.

10. Merezhinskaya N. Fishbein WN, Davis JI, et al. Mutations in MCT1 cDNA in patients with symptonatic deficiency in lactate transport. Muscle Nene 2000;23(January (1));90-7.

11. Knuttgen HG. Strength training and aerobic exercise: comparison and contrast. J Strength Cond Res 2007;21(Auguat (3)):973-8.

12. Kang J. Hoffman JR, Im J. et al. Evaluation of physiological responses during recovery following three resistance exercise prograns. I Strength Cond Res 2005;19(May (2)):305-9.

13. Scott CB. Contribution of blood lactate to the energy expenditure of weight training. J Strength Cond Res 2006;20(May (2)):404-11.

14. Carter L, Heath B. Somatotyping. Development and applications. Canbridge: University Press; 1990.
15. Martin AD, Spenst LF. Drinkwater DT, et al. Anthropometric estimation of muscle mass in men. Med Sci Sports Exerc 1990;22(October (5)):729-33.

16. Willardson JM, Burkett LN. The effect of rest interval length on the sustainability of squat and bench press repetitions. I Strength Cond Res 2006;20(May (2)):400-3.

17. Kraemer WJ, Ratamess NA. Hormonal responses and adaptations to resistance exercise and training. Sports Med 2005;35(4):339_ 61 .

18. Bonen A, McCullagh KJ, Putman CT, et al. Short-term training increases human muscle MCT1 and femoral venous lactate in relation to nuscle lactate. Am J Physiol 1998:274(January (1 Pt 1)):El02-7.

19. Dubouchaud H. Butterfield GE. Wolfel EE, et al. Endurance training. expression, and physiology of LDH, MCT1, and MCT4 in human skeletal muscle. Am J Physiol Endocritol Metab 2000;278(April (4)): $\mathrm{E} 571-9$.

20. Bickham DC. Bentley DJ. Le Rossignol PF. et al. The effects of shott-term sprint training on MCT expression in moderately endurance-trained runners. Eur J Appl Physiol 2006;96(April (6)): $636-43$.

21. Burgonaster KA, Cermak NM. Phillips SM. et al. Divergent response of metabolite transport proteins in human skeletal nuscle after sprint interval training and detraining. Am J Physiol Regul Integr Comp Physiol 2007;292(May (5)):R 1970-6.

22. Juel C. Klarskov C, Nielsen JJ, et al. Effect of high-intensity intermittent training on lactate and $\mathrm{H}+$ release from human skeletal nuscle. $A m$ J Physiol Endocrinol Metab 2004;286(February (2)):E245-51.

23. Pilegaard H, Terzis G. Halestrap, et al. Distribution of the lactate/H+ transporter isoforms MCTl and MCT4 in buman skeletal muscle. Am J Physiol 1999;276(May (5 Pt 1)):E843-8.

24. Bishop D, Edge J, Thomas C, et al. High-intensity exercise acutely decreases the membrane content of MCT1 and MCT4 and buffer capacity in human skeletal nuscle. $J$ Appl Physiol 2007:102(February (2)):616-21.

25. Gladden LB. Muscle as a consumer of lactate. Med Sci Sports Exerc 2000;32(April (4)):764-71.

26. Chen YJ, Wong SH, Wong CK, et al. Effect of preexercise meals with different glycenic indices and loads on metabolic responses and endurance running. Int $J$ Sport Nutr Exer Metab 2008;18(June (3)) $: 281-300$

27. Stevenson E. Willians C. Nute M. The influence of the glycaemic index of breakfast and lunch on substrate utilization during the postprandial periods and subsequent exercise. BrJ Nutr 2005;93(June (6)): $885-93$.

28. Wu CL, Nicholas $\mathrm{C}$, Williams $\mathrm{C}$, et al. The influence of highcarbohydrate meals with different glycaenic indices on substrate utilization during subsequent exercise. Br J Nutr 2003;90(December (6)):1049-56. 